This is a postprint version of the following document:

L. Leyva, J. Pinheiro, D. Castanheira, A. Silva, and A. Gameiro, "Alamouti coding scheme and virtual array concept for joint radar and communication systems," 12th IEEE/IET International Symposium on Communication Systems, Networks and Digital Signal Processing, (CSNDSP), 20-22, July 2020 (Online), 4 pp.

(C) 2020 IEEE. Personal use of this material is permitted. Permission from IEEE must be obtained for all other uses, in any current or future media, including reprinting/republishing this material for advertising or promotional purposes, creating new collective works, for resale or redistribution to servers or lists, or reuse of any copyrighted component of this work in other works. 


\title{
Alamouti coding scheme and virtual array concept for joint radar and communication systems
}

\author{
L. Leyva*, J. Pinheiro ${ }^{\dagger}$, D. Castanheira ${ }^{\ddagger}$, A. Silva*, and A. Gameiro* \\ ${ }^{*}$ Instituto de Telecomunicações (IT) and DETI, University of Aveiro, Portugal \\ Email: \{leoleval, asilva\}@av.it.pt, and amg@ua.pt \\ ${ }^{\dagger}$ DETI, University of Aveiro, Portugal \\ Email: jmaciel@ua.pt \\ ${ }^{\ddagger}$ Instituto de Telecomunicações (IT), Portugal \\ Email: dcastanheira@av.it.pt
}

\begin{abstract}
This paper focuses on the integration of radar and communication (RadCom) systems on a common platform. We consider a scenario with multiple transmit and receive antennas, where the integration of the two functionalities is achieved through the use of Alamouti coding and OFDM waveform. It is shown that by using the Alamouti coding scheme, the spatial diversity order is improved as in legacy communication systems, which has a favorable impact on the bit error rate (BER). Furthermore, as the Alamouti code is an orthogonal code the radar's angle resolution is improved through the use of the virtual array concept. The developed RadCom system presents good performance, low complexity and, therefore, is of interest for practical applications.
\end{abstract}

Index Terms-Alamouti coding, OFDM, Radar, Communication, RadCom, convergence, MIMO

\section{INTRODUCTION}

Wireless communication and radar systems have been historically designed and developed in total isolation from one another. Nevertheless, they share similar properties that can facilitate systems handling the trend towards less exclusionary spectrum policies and interference [1]. Nowadays, the scarcity of the radio spectrum is becoming a problem for the telecommunication sector, leading to the study of new manners for coexistence [2]. Research of joint radar and communication (RadCom) systems arise as one of the candidate paradigms seeking to make more efficient use of the radio spectrum, relieving the scarcity problem.

The idea of combining these two systems in one is not new. However, the existence of such systems is rare. NASA Space Shuttle "Orbiter" is one of the few examples, the system could switch between radar and communication but not perform both at the same time [3]. Finding a suitable waveform is a central challenge to achieve functional joint radar and communications systems. Several approaches rely on radar waveforms [4] [5] to converge both functionalities into a single platform. These schemes share the drawback of low data rates for the communication function as shown in [6], where data rates are limited to $100 \mathrm{kbps}$, which corresponds to the pulse repetition frequency (PRF) only.

This work has received funding from the European Union's Horizon 2020 research and innovation programme under the Marie Skłodowska-Curie ETN TeamUp5G, grant agreement No. 813391.
Other approaches rely on the use of communication waveforms to achieve such a convergence. In [7], the direct sequence spread spectrum (DSSS) waveform is employed, taking advantage of its good correlation properties, while [8] represents the first attempt to use orthogonal frequencydivision multiplexing (OFDM) waveform for RadCom systems. Data dependency, which is inherent to the OFDM waveform, negatively influences the correlation properties of this waveform, which can be catastrophic for proper targets unambiguous ranging. A huge step seeking to relieve this effect was taken in [9], where the data dependency is removed by performing an element-wise complex division between the received signal and the transmitted symbols. More recently, [10] addresses the principles of OFDM waveform for radar processing, with particular focus on the Long Term Evolution (LTE) networks. Spectrum scarcity and the constant need for higher bandwidth has led to the study of new waveforms, this time in the millimeter-wave (mmWave) part of the spectrum as in [11] [12].

A system performing both functionalities on the same hardware platform can be beneficial in terms of cost, dimension, performance and spectral efficiency. Besides, the integration of wireless communication and radar sensing systems will bring up many benefits in areas such as intelligent transportation systems (ITS) [13], wireless sensor networks (WSN), Internet of Things (IoT) [14] and modern military applications.

This paper considers a scenario with a monostatic RadCom terminal transmitting information, using mmWave carrier, towards one user's equipment [15]. The integration of radar and communication systems is accomplished by using OFDM as a common waveform and the use of Alamouti coding enables both the achievement of spatial diversity, for the communication functionality, and of transmit signal orthogonality required to improve resolution, for the radar functionality.

The remainder of this paper is organized as follows: Section II defines the system model for the RadCom transmitter, communication device processing and the radar processing required to estimate target parameters. Section III is devoted to the performance results for RadCom systems regarding BER and radar imaging. Finally, section IV concludes the proposal addressed in this paper. 


\section{SYSTEM MODEL}

As illustrated in Fig. 1, we consider a RadCom access point transmitting a common waveform. The signal transports communication information to the mobile device equipped with a receiving antenna.

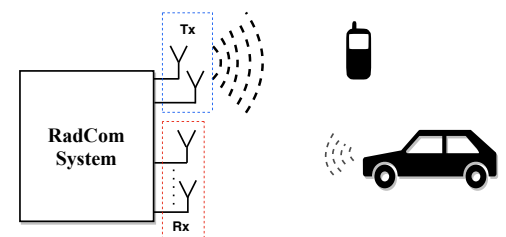

Fig. 1. RadCom System scenario.

At the same time, the signal is reflected from targets within the coverage area. The RadCom access point receives the echoes of its own transmit signal and detects the presence of targets. For communication processing, the modulation type is perfectly known to the communication terminal. For radar processing, the transmitted signal is known at the radar receiver. Two antennas are transmitting the same information, seeking orthogonality of the waveforms, before the transmission, the data is encoded by using the Alamouti coding to allow joint radar and communications functionalities.

\section{A. RadCom Transmitter}

Consider a RadCom system with two transmitting antennas, spacing by a distance of $Q \lambda / 2$, where $Q$ represents the number of receiving antennas and $\lambda$ the wavelength. To enhance the diversity order achieved, we consider the known Alamouti space-time scheme, coding the OFDM waveform in the time domain. Fig. 2 shows the block diagram of the RadCom transmitter implemented.

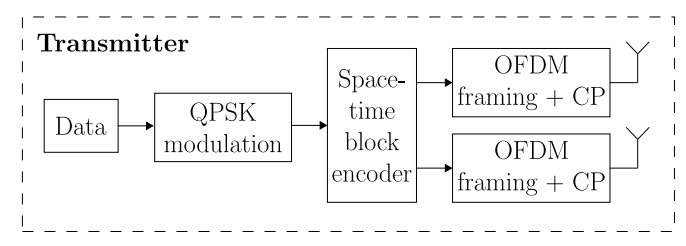

Fig. 2. Block diagram of the RadCom Transmitter.

The binary data stream is first modulated and mapped from a QPSK modulation alphabet into a sequence of symbols and divided in two data streams $\mathbf{c}_{1}$ and $\mathbf{c}_{2}$.

The Alamouti code is performed over a block with two time slots. The coding matrix for subcarrier $k$ and block $l$ is

$$
\mathbf{C}_{k, l}=\left[\begin{array}{cc}
\mathbf{c}_{1}(k, l) & \mathbf{c}_{2}(k, l) \\
-\mathbf{c}_{2}^{*}(k, l) & \mathbf{c}_{1}^{*}(k, l)
\end{array}\right],
$$

with $k=\{0, \cdots, N-1\}, l=\{0, \cdots, M-1\}$. Column 1 (2) of matrix $\mathbf{C}_{k, l}$ corresponds to the signal to be transmitted on antenna 1 (2). After the STBC block, an IFFT operation is performed, then a cyclic prefix $(\mathrm{CP})$ is added to avoid intersymbol interference (ISI).

\section{B. Communication terminal}

Consider a communication device with one receiving antenna located within the coverage area of the RadCom transmitter. We aim to recover the original data sent from the RadCom system. Fig. 3 illustrates the scheme of the communication terminal considered.

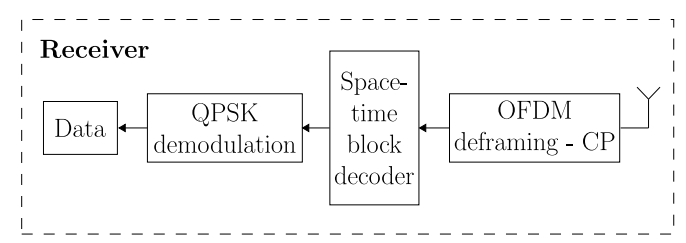

Fig. 3. Alamouti scheme with 1 receive antenna.

Assuming the coherence time of the channel is higher than the duration of two consecutive OFDM symbols, the received signal model at the communication device for two consecutive symbols after removing the $\mathrm{CP}$ and performing the FFT operation can be expressed as

$$
\mathbf{r}_{k, l}=\mathbf{C}_{k, l} \mathbf{h}_{k, l}+\mathbf{n}_{k, l}
$$

where $\mathbf{r}_{k, l}$ denotes the OFDM received signal, $\mathbf{h}_{k, l}$ the channel frequency response between the two transmitting antennas and the single receiving antenna and $\mathbf{n}_{k, l} \sim \mathcal{C N}\left(0, N_{0} \mathbf{I}\right)$ denotes white Gaussian noise. The soft decision of $\mathbf{c}_{k, l}=$ $\left[\mathbf{c}_{1}(k, l), \mathbf{c}_{2}(k, l)\right]^{T}$ is obtained by performing the operation

$$
\tilde{\mathbf{c}}_{k, l}=\mathbf{H}_{k, l}^{H} \overline{\mathbf{r}}_{k, l}
$$

where $\mathbf{H}_{k, l}=\left[\mathbf{h}_{k, l}(1), \mathbf{h}_{k, l}(2) ; \mathbf{h}_{k, l}^{*}(2),-\mathbf{h}_{k, l}^{*}(1)\right]$ and $\overline{\mathbf{r}}_{k, l}=$ $\left[\mathbf{r}_{k, l}(1), \mathbf{r}_{k, l}^{*}(2)\right]^{T}$.

Substituting (2) into (3), the next relation is obtained, where is easy to see that the ISI is completely removed,

$$
\tilde{\mathbf{c}}_{k, l}=\left\|\mathbf{h}_{k, l}\right\|^{2} \mathbf{c}_{k, l}+\mathbf{H}_{k, l}^{H} \mathbf{n}
$$

The equality (4) follows from the equality $\mathbf{H}_{k, l}^{H} \mathbf{H}_{k, l}=\left\|\mathbf{h}_{k, l}\right\|^{2} \mathbf{I}$

\section{Radar terminal}

Consider a radar configuration consisting of a transmit antenna array composed of two antennas and a receive uniform antenna array with $Q$ antennas with an inter-antenna distance of $\lambda / 2$. The received signal model at the $q$-th receiving antenna, for sub-carrier $k$ and block $l$, is given by

$$
\mathbf{r}_{k, l}^{q}=\mathbf{C}_{k, l} \mathbf{h}_{k, l}^{q}+\mathbf{n}_{k, l}
$$

where $\mathbf{r}_{k, l}^{q}$ denotes the OFDM received signal, $\mathbf{h}_{k, l}^{q}$ the channel frequency response between the two transmitting antennas and the single receiving antenna and $\mathbf{n}_{k, l} \sim \mathcal{C N}\left(0, N_{0} \mathbf{I}\right)$ denotes white Gaussian noise.

At the radar terminal the objective is to recover the channel matrix $\mathbf{H}_{k, l}=\left[\mathbf{h}_{k, l}^{1}, \cdots, \mathbf{h}_{k, l}^{Q}\right]$. The channel relative to antenna $q$ may be estimated as,

$$
\begin{aligned}
\tilde{\mathbf{h}}_{k, l}^{q} & =\mathbf{C}_{k, l}^{H} \mathbf{r}_{k, l}^{q} \\
& =\mathbf{h}_{k, l}^{q}+\mathbf{C}_{k, l}^{H} \mathbf{n}_{k, l}^{q}
\end{aligned}
$$


where the last equality follows from $\mathbf{C}_{k, l}^{H} \mathbf{C}_{k, l}=\mathbf{I}$. Therefore, follows that

$$
\begin{aligned}
\tilde{\mathbf{H}}_{k, l} & =\mathbf{C}_{k, l}^{H} \mathbf{R}_{k, l} \\
& =\mathbf{H}_{k, l}+\mathbf{C}_{k, l}^{H} \mathbf{N}_{k, l}
\end{aligned}
$$

where $\mathbf{R}_{k, l}=\left[\mathbf{r}_{k, l}^{1}, \cdots, \mathbf{r}_{k, l}^{Q}\right]$ and $\mathbf{N}_{k, l}=\left[\mathbf{n}_{k, l}^{1}, \cdots, \mathbf{n}_{k, l}^{Q}\right]$.

Let us define the vector $\mathbf{a}_{k, l}=\operatorname{vec}\left(\mathbf{H}_{k, l}^{T}\right)$, where the operator vec(.) vectorizes the input matrix column by column, then assuming a target at an azimuth angle of $q$, a range $R$ and a speed $v$ the entry $p \in\{0, \cdots, 2 Q-1\}$ of vector $\mathbf{a}_{k, l}$ is

$$
\mathbf{a}_{k, l}(p)=e^{j 2 \pi T_{0} f_{D} l} e^{-j 2 \pi k \Delta f \tau} e^{-j 2 \pi p \phi}
$$

where $f_{D}=2 v / \lambda$ denotes the Doppler frequency, $\tau=2 R / c_{0}$ the delay, $\phi=\sin (\theta)$ the electrical angle, $T_{0}$ the OFDM symbol duration, $\Delta f$ the sub-carrier spacing and $c_{0}$ the speed of light. Therefore, from $\tilde{\mathbf{H}}_{k, l}$ follows $\tilde{\mathbf{a}}_{k, l}=\operatorname{vec}\left(\tilde{\mathbf{H}}_{k, l}^{T}\right)$ where entry $p$ is $\tilde{\mathbf{a}}_{k, l}(p)$. The channel response $\mathbf{a}_{k, l} \in \mathbb{C}^{2 Q}$ is identical to the channel response of a system with one transmitting antenna and a uniform linear array with $2 Q$ receiving antennas with an inter-antenna distance of $\lambda / 2$. This $2 Q$ - element array is the virtual antenna array, which is obtained with just $2+Q$ physical antenna elements.

Accordingly to (8) the target parameters may be estimated by performing an DFT or IDFT along the three dimensions $(k, l, p)$. The IDFT performed along the $k$ dimension provides an estimative of the range, the DFT along the $l$ dimension an estimative of the velocity and and the IDFT along the $p$ dimension provides an estimative of the electrical angle (target angle).

\section{Performance Results}

In this section, the performance of the RadCom system proposed is evaluated. The limiting properties of the channel are the Doppler spread and the maximum delay. The radar cross-section (RCS) of targets is not taken into account. For this system, we present the average bit error rate (BER) performance and a radar image obtained for one target. Consider a RadCom system with 2 transmitting antennas and 10 receiving antennas. There are considered two scenarios with the parameters described in Table I.

TABLE I

TARGET PARAMETERS

\begin{tabular}{c|c|c} 
Scenario & Target's angle & Velocity \\
\hline \hline $\mathrm{A}$ & $30^{\circ}$ & $100 \mathrm{~m} / \mathrm{s}$ \\
\hline $\mathrm{B}$ & $70^{\circ}$ & $100 \mathrm{~m} / \mathrm{s}$
\end{tabular}

The system parameters are chosen to fulfill a set of design criteria as, maximum unambiguous range $R$ and range resolution $\Delta r$. The system operates at $f_{c}=24 \mathrm{GHz}$ ISM mmWave band, which has been used for research purposes as it is deregulated. To be able to detect targets in at least 1500 $\mathrm{m}$, the maximum unambiguous range is defined by

$$
R=\frac{c_{0}}{2 \Delta f}=\frac{T c_{0}}{2},
$$

where $T$ denotes the OFDM symbol duration, defining $T=$ $11 \mu$ s a maximum unambiguous range of $R=1650 \mathrm{~m}$ is obtained. The range resolution is given by

$$
\Delta r=\frac{c_{0}}{2 B}
$$

where $B$ denotes the system bandwidth, since we need to ensure high data rates and good range resolution, the number of sub-carriers is set to $N=1024$. Since $\Delta f=1 / T$, a bandwidth of $B=93.1 \mathrm{MHz}$ is required, resulting in a range resolution of $\Delta r=1.61 \mathrm{~m}$. These and other radar parameters are summarized in Table II.

TABLE II

RADAR SPECIFICATIONS: RADCOM SYSTEM.

\begin{tabular}{l|c} 
Unambiguous range $R$ & $1650 \mathrm{~m}$ \\
\hline Range resolution $\Delta r$ & $1.61 \mathrm{~m}$ \\
\hline Velocity Resolution $\Delta v$ & $2.22 \mathrm{~m} / \mathrm{s}$ \\
\hline Angle Resolution $\Delta \Theta$ & $3.8^{\circ}$ \\
\hline Subcarriers spacing $\Delta f$ & $90.09 \mathrm{kHz}$ \\
\hline Number of OFDM symbols $M$ & 256
\end{tabular}

Fig. 4 and 5 shows the radar imaging obtained for one target for the scenarios described in Table I.

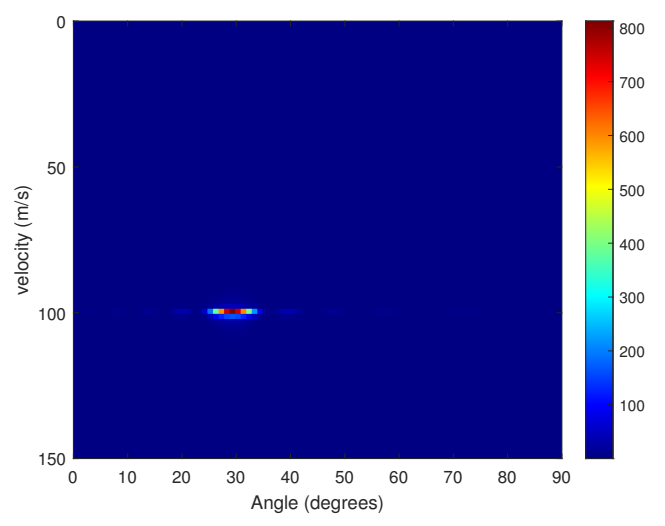

Fig. 4. Radar imaging obtained for scenario $\mathbf{A}$ and Radar Specifications in Table II.

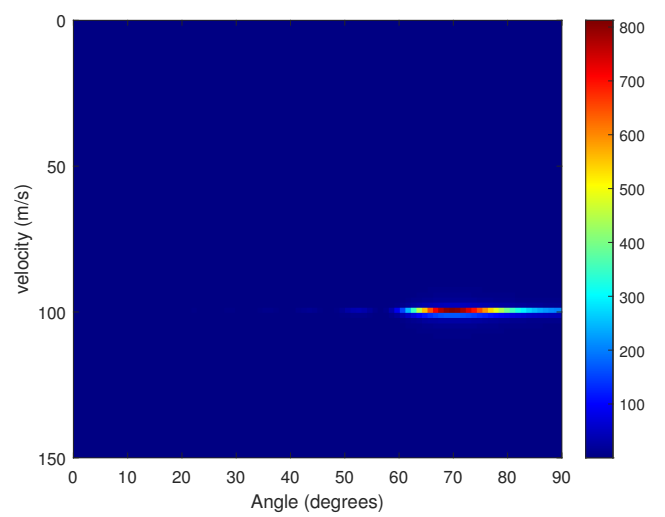

Fig. 5. Radar imaging obtained for scenario $\mathbf{B}$ and Radar Specifications in Table II 
The results obtained in Fig. 4 and 5 show that as the angle where the object is positioned increases, the image resolution deteriorates. Fig. 6 shows the BER at the communication terminal.

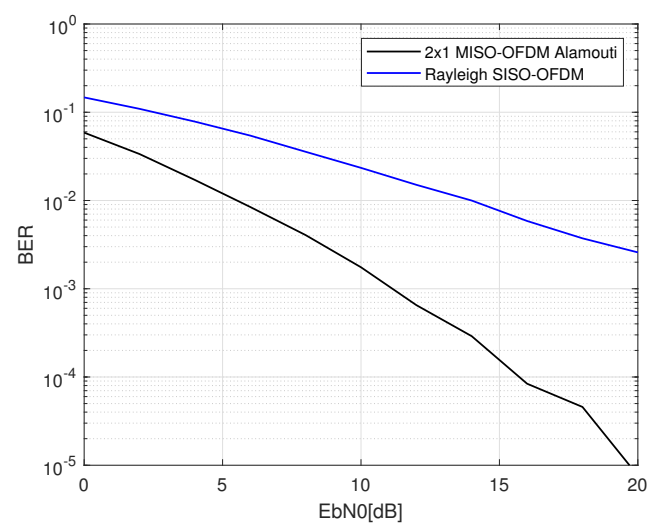

Fig. 6. BER perfomance evaluation of Rayleigh fading channel and 2x1 MISO-OFDM Alamouti at the communication terminal device.

From Fig. 6 is possible to see that as in legacy wireless communication systems the use of two antennas transmitting orthogonal signals bring with an improving in the BER performance. As can be noticed, the slope of 2x1 MISO-OFDM Alamouti is larger than that one of Rayleigh SISO-OFDM, this is due to the diversity gain of 2 that $2 \times 1$ MISO-OFDM Alamouti system can achieve compared to SISO system that has a diversity order of 1 .

\section{CONCLUSIONS}

In this paper, the integration of radar and communication systems is accomplished by using OFDM as a common waveform and the use of Alamouti coding schemes. Alamouti code enables both the achievement of spatial diversity, for the communication functionality, and improve angle resolution for the radar functionality, using the virtual antenna array concept. The flexible allocation of carriers was one of the motivation for choosing OFDM as the the most suitable waveform. These scenarios represent the basis for future research, in which the use of more than two transmitting antennas can be accomplished by using other codes, even going to the implementation of massive multiple-input multiple-output (mMIMO).

\section{REFERENCES}

[1] A. Garnaev, W. Trappe and A. Petropulu, "Bargaining over fair performing dual radar and communication task," 2016 50th Asilomar Conference on Signals, Systems and Computers, Pacific Grove, CA, 2016, pp. 47-51.

[2] L. Zheng, M. Lops, Y. C. Eldar and X. Wang, "Radar and Communication Coexistence: An Overview: A Review of Recent Methods," in IEEE Signal Processing Magazine, vol. 36, no. 5, pp. 85-99, Sept. 2019.

[3] R. Cager, D. LaFlame and L. Parode, "Orbiter Ku-Band Integrated Radar and Communications Subsystem," in IEEE Transactions on Communications, vol. 26, no. 11, pp. 1604-1619, November 1978.

[4] G. N. Saddik, R. S. Singh and E. R. Brown, "Ultra-Wideband Multifunctional Communications/Radar System," in IEEE Transactions on Microwave Theory and Techniques, vol. 55, no. 7, pp. 1431-1437, July 2007.
[5] S. D. Blunt, P. Yatham and J. Stiles, "Intrapulse Radar-Embedded Communications," in IEEE Transactions on Aerospace and Electronic Systems, vol. 46, no. 3, pp. 1185-1200, July 2010.

[6] J. Kota, C. Topliff, R. Prasanth, G. Ushomirsky and S. Kogon, "RF Convergent Waveform Design Using Time-Modulated Phase Functions," 2018 52nd Asilomar Conference on Signals, Systems, and Computers, Pacific Grove, CA, USA, 2018, pp. 409-413.

[7] X. Shaojian, C. Bing and Z. Ping, "Radar-Communication Integration Based on DSSS Techniques," 2006 8th international Conference on Signal Processing, Beijing, 2006

[8] B. J. Donnet and I. D. Longstaff, "Combining MIMO Radar with OFDM Communications," 2006 European Radar Conference, Manchester, 2006 pp. $37-40$.

[9] C. Sturm and W. Wiesbeck, "Waveform Design and Signal Processing Aspects for Fusion of Wireless Communications and Radar Sensing," in Proceedings of the IEEE, vol. 99, no. 7, pp. 1236-1259, July 2011.

[10] C. B. Barneto, L. Anttila, M. Fleischer and M. Valkama, "OFDM Radar with LTE Waveform: Processing and Performance," 2019 IEEE Radio and Wireless Symposium (RWS), Orlando, FL, USA, 2019, pp. 1-4.

[11] K. V. Mishra, M. R. Bhavani Shankar, V. Koivunen, B. Ottersten and S. A. Vorobyov, "Toward Millimeter-Wave Joint Radar Communications: A Signal Processing Perspective," in IEEE Signal Processing Magazine, vol. 36, no. 5, pp. 100-114, Sept. 2019.

[12] F. Liu and C. Masouros, "Hybrid Beamforming with Sub-arrayed MIMO Radar: Enabling Joint Sensing and Communication at mmWave Band," ICASSP 2019 - 2019 IEEE International Conference on Acoustics, Speech and Signal Processing (ICASSP), Brighton, United Kingdom, 2019, pp. 7770-7774.

[13] P. Kumari, N. Gonzalez-Prelcic and R. W. Heath, "Investigating the IEEE 802.11 ad Standard for Millimeter Wave Automotive Radar," 2015 IEEE 82nd Vehicular Technology Conference (VTC2015-Fall), Boston, MA, 2015, pp. 1-5.

[14] Z. Khan, J. J. Lehtomaki, S. I. Iellamo, R. Vuohtoniemi, E. Hossain and Z. Han, "IoT Connectivity in Radar Bands: A Shared Access Model Based on Spectrum Measurements," in IEEE Communications Magazine, vol. 55, no. 2, pp. 88-96, February 2017.

[15] B. Paul, A. R. Chiriyath and D. W. Bliss, "Survey of RF Communications and Sensing Convergence Research," in IEEE Access, vol. 5, pp. 252-270, 2017. 